\title{
Effects of Glucomannan Noodle on Diabetes Risk Factors in Patients with Metabolic Syndrome: A Double-Blinded, Randomized Crossover Controlled Trial
}

\author{
Kuan-Un Cheang ${ }^{1}$, Chiao-Ming Chen ${ }^{2}$, C-Y. Oliver Chen ${ }^{3}$, Feng-Yu Liang ${ }^{4}$, Chun-Kuang Shih ${ }^{1}$, Sing-Chung Li ${ }^{1, *}$ \\ ${ }^{1}$ School of Nutrition and Health Science, College of Nutrition, Taipei Medical University, Taipei, Taiwan \\ ${ }^{2}$ Department of Food Science, Nutrition, and Nutraceutical Biotechnology, Shih Chien University, Taipei, Taiwan \\ ${ }^{3}$ Antioxidants Research Laboratory, Jean Mayer USDA Human Nutrition Research Center on Aging, Tufts University, Boston, MA \\ ${ }^{4}$ Department of Hospitality Management, Hsuan Chuang University, Hsinchu, Taiwan \\ *Corresponding author: sinchung@tmu.edu.tw
}

\begin{abstract}
Glucomannan is a water-soluble dietary fiber derived from the root of Amorphophallus konjac that can improve blood sugar, blood fat concentration, and weight management, and has other health benefits. The aim of this study is to investigate the effect of glucomannan noodles on components of the metabolic syndrome. A randomized, double-blind, placebo-controlled, crossover study, 32 individuals with metabolic syndrome were received a daily servings of 400 gram glucomannan noodles for a period of four weeks. After two weeks of wash out period, they received a placebo noodles for four weeks. There were no statistical difference in calories, carbohydrate, protein, fat and dietary fiber intake from $24 \mathrm{~h}$ food recalls between glucomannan noodles and placebo noodles groups. However, the body weight, body mass index, and waist circumference had significantly decreased after 4-week intervention in both groups, but high sensitivity C-reactive protein was lowered only in glucomannan noodles group. Moreover, the 24 individuals with type 2 diabetes had significantly decreased body weight, BMI, waist circumference, hs-CRP, and glycated hemoglobin after glucomannan noodles intervention. The rest of lipid profile, fasting blood glucose, insulin, and HOMA-IR of the subjects did not show any significant difference. This study showed that glucomannan noodles as a staple food can contribute to metabolic syndrome in adults predisposed to type 2 diabetes.
\end{abstract}

Keywords: konjac noodle, metabolic syndrome, diabetes mellitus

Cite This Article: Kuan-Un Cheang, Chiao-Ming Chen, C-Y. Oliver Chen, Feng-Yu Liang, Chun-Kuang Shih, and Sing-Chung Li, "Effects of Glucomannan Noodle on Diabetes Risk Factors in Patients with Metabolic Syndrome: A Double-Blinded, Randomized Crossover Controlled Trial.” Journal of Food and Nutrition Research, vol. 5, no. 8 (2017): 622-628. doi: 10.12691/jfnr-5-8-13.

\section{Introduction}

Metabolic syndrome (MS) is a complex disease with a cluster of risk factors and clinical features, including central or abdominal obesity, atherogenic dyslipidemia, impaired glucose regulation with compensated hyperinsulinemia, high blood pressure, and concomitant proinflammatory cytokine and insulin resistance. [1,2] Central obesity induced insulin resistance and dyslipidemia caused by atherosclerosis, which is considered as two main pathogenesis with a chronic inflammatory for MS, however no single treatment for the syndrome as a whole therapeutic approaches.[3] Patients with MS have an increased risk of developing type 2 diabetes mellitus (T2DM), which is strongly associated with the presence of abdominal obesity [4]. The main goal for managing MS is preventing atherosclerotic cardiovascular disease and delay the onset of T2DM. [4,5] The intake of foods containing whole grains and dietary fiber (DF) has been associated with improved insulin sensitivity for most patients with diabetes. [6,7] According to the Standard Care of Diabetes from the American Diabetes Association, early lifestyle intervention and medical nutrition therapy are highly recommended for management of MS, and DF intake at $14 \mathrm{~g} / 1000 \mathrm{kcal} /$ day is suggested ( $>25 \mathrm{~g}$ for women and $>38 \mathrm{~g}$ for men is the daily recommendation), but the actual average daily intake for Americans has been less than half of the daily recommendation in the past 10 years, [8] and a lower intake of DF was observed to increase the incidence of T2DM in Taiwanese people. [9] A cross-sectional study conducted by Steemburgo et al. showed that the intake of soluble DF from whole grains and fruits can have protective effects for MS patients with T2DM. [7]

Amorphophallus konjac (konjac) has long been used in China, Japan, and Southeast Asia as a food source and traditional medicine. Konjac flour extracted from the corm of this species is used to make noodles, tofu, and snacks. 
[10] Crude konjac mannan in an alkalinized, gelled form is a traditional foodstuff eaten as a normal part of the diet. [11] Glucomannan is a $100 \%$ water-soluble DF that is derived mainly from the tuberous roots of $A$. konjac. It is a compound of straight chain polysaccharides with $\beta-(1-4)$ D-mannose and D-glucose units in a ratio of 1.6:1, with a small amount $(8 \%)$ of branching through $\beta-(1-6)$ glucocyl linkage. The main component, glucomannan, has an average molecular weight of 200 to $2000 \mathrm{kDa}$ and cannot be hydrolyzed by salivary and pancreatic amylase. [10] Chen et al. investigated the effect of glucomannan supplementation and found that $1.5 \mathrm{~g}$, three times a day, could be an optimum dosage to promote defecation frequency primarily from the increased stool bulk in constipated adults. [12] In a study conducted by Yoshida et al., the effect of glucomannan (10 g/day) plus plant sterols (1.8 g/day) reduced plasma low density lipoprotein cholesterol (LDL-C) concentrations in subjects with T2DM after 21 days of intervention. [13]

The European Food Safety Authority confirmed the positive effect of glucomannan on the reduction of body weight (BW) in overweight adults in 2010 when a daily supplement of $3 \mathrm{~g}$ of glucomannan proved helpful in reducing BW in obese or overweight adults. [14] glucomannan could also be used as a laxative in the treatment of childhood constipation [15] and has a prebiotic function that selectively enhances the growth of probiotic bacteria such as lactobacilli and bifidobacteria. [16] A meta-analysis by Sood et al. showed significantly lowered total cholesterol, triglycerides, BW, and fasting blood glucose (FBG) after glucomannan intervention. [17] A konjac-derived glucomannan supplement (3.6 g/day) administered for 28 days reduced blood lipid and glucose levels by enhancing fecal excretion of neutral sterol and bile acid and alleviated the elevated glucose levels in hyperglycemic diabetic subjects. [18] As far as we know, no studies have evaluated the influence of konjac-derived glucomannan noodle as staple in patients with metabolic syndrome. We hypothesized that who consumed 400 gram glucomannan noodle daily for four weeks would show beneficial changes from baseline in serum factors for metabolic syndrome. Our specific aim was to examine the effect of glucomannan noodle on glucoregulation, lipid metabolism and inflammation in individuals with metabolic syndrome.

\section{Materials and Methods}

\subsection{Subjects}

All participants had been diagnosed with MS according to the inclusion criteria from the National Cholesterol Education Program Adult Treatment Panel III 2004 [19] for at least 6 months, and some were receiving regular therapy. The average ages were $53.6 \pm 7.9$ years for men and $51.4 \pm 12.3$ for women. The baseline body height was significantly higher for the men, among whom smoking and drinking habits were also predominant. Any cases involving insulin dependency, the use of oral or injected steroids, or the abuse of hypnotics or other drugs were excluded. Anyone with acute illnesses, such as recent myocardial infarction, major surgery, upper or lower gastrointestinal tract bleeding, or poor control of blood glucose and blood pressure, was excluded from the clinical trial. Patients with malignancy, endocrine diseases other than diabetes, congestive heart failure, liver cirrhosis, renal insufficiency with serum creatinine $(\mathrm{Cr})$ greater than $3.0 \mathrm{mg} / \mathrm{dL}$, or moderate anemia with hemoglobin less than $9 \mathrm{~g} / \mathrm{dL}$ were also excluded, as were pregnant or lactating women. Ultimately, 32 patients with MS (16 women, 16 men) underwent the 4-week GMN to placebo noodle (PN) or PN to GMN interventions. Of these patients, 24 (14 women, 10 men) had T2DM concomitant with MS.

All participants provided basic information about anthropometric measurements, BW, body height, BMI, waist circumference, waist-hip ratio, blood pressure, and pulse rate. They also supplied the following personal information: family medical history, physical activity level, educational level, working status, smoking and alcohol habits, tea and milk drinking habits, and 24-hour dietary recall. Before participating in the study, the subjects were offered the opportunity to discuss any queries with the primary investigator, the physician, and the study coordinator. All subjects provided informed written consent, and both anthropometric measurements and personal information were collected. The study protocol was approved by the Institutional Review Board of the Taipei Medical University, and written consent was obtained from participants before study procedures were performed. The study was registered at ClinicalTrials.gov (NCT02711306).

\subsection{Design}

The study was a four weeks randomized, crossover, controlled feeding trial with a two weeks run-in period and a two weeks washout between alternative diets (Figure 1). We enrolled 40 individuals diagnosed with MS according to the inclusion criteria in the trials, although four individuals were excluded for not meeting entry criteria. Thirty-six subjects were randomly assigned to the GMN/PN or PN/GMN sequence with a 2-week washout period between alternative diets. Two individuals were withdrew due to taking a probiotic supplement and migration in the GMN/PN sequence. Another two individuals were excluded due to catching a flu and taking anti-inflammatory drug in the PN/GMN sequence. In the GMN diet, the participants received two servings ( $400 \mathrm{~g}$ ) of GMNs every day to replace their daily carbohydrate intake for 4 weeks, with each serving of glucomannan noodles weighing up to $200 \mathrm{~g}$ with $2 \mathrm{~g}$ of glucomannan. In the PN diet, the participants received the same amount of noodles without glucomannan. After the 4-week intervention period, a 2week washout period was observed to let the participants revert to their original meal habits. Then, the GMN diet and PN diet were switched during the crossover stage for a further 4 weeks. Total 32 MS subjects, including 24 with T2DM, were analyzed at the end of the trial. All subjects were encouraged not to otherwise change their ordinary diets or general lifestyle during the study. To make sure the compliance of their diets and physical activities during the trial, they were asked to record their food intake and physical activities weekly during intervention period. 


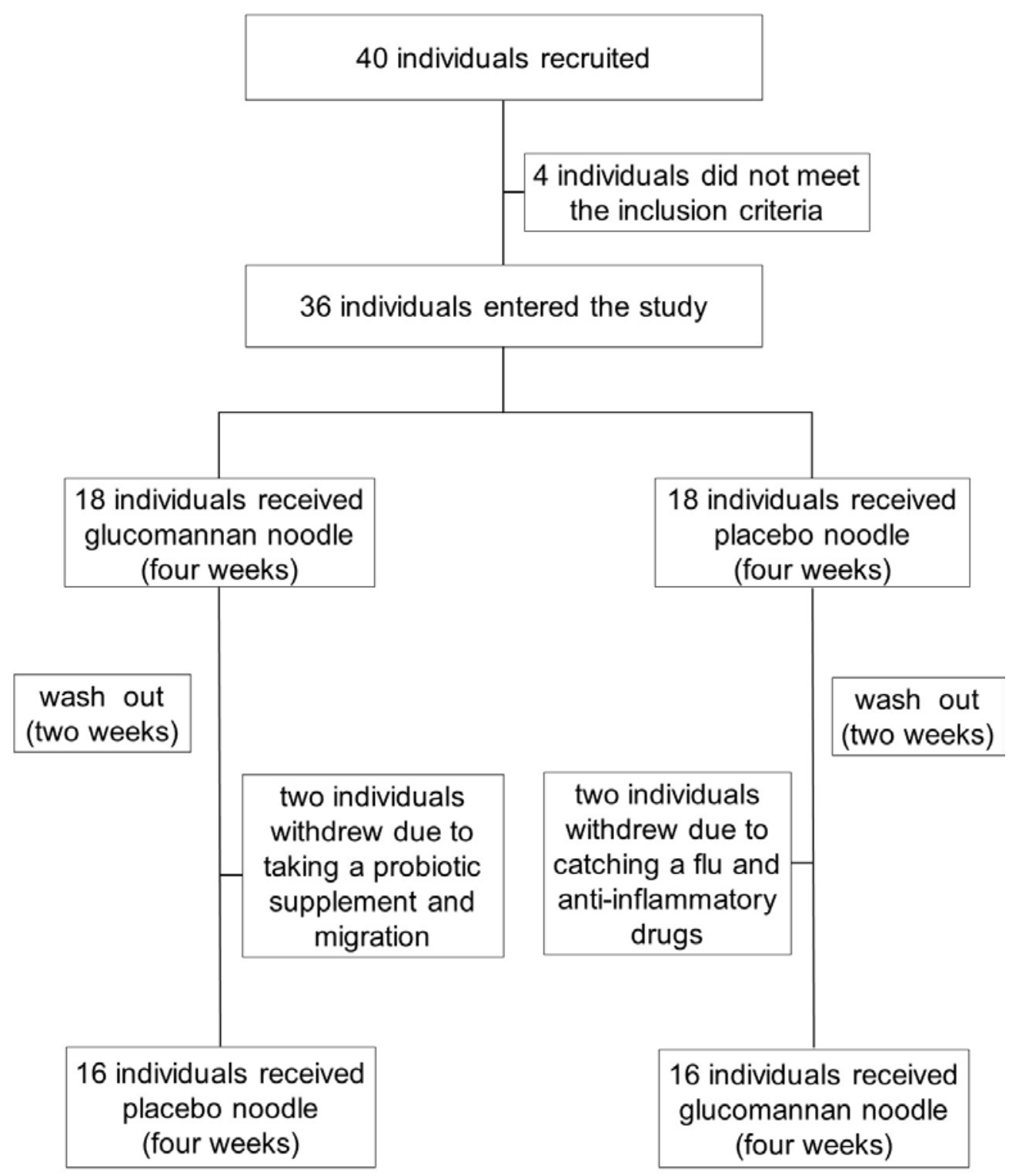

Figure 1.

\subsection{Composition of Glucomannan Noodles}

In this study, each serving of noodles weighed $200 \mathrm{~g}$ (one serving). The $100 \mathrm{~g}$ GMN contains 159 kilocalories, $1 \mathrm{~g}$ of konjac-derived glucomannan powder, $7.9 \mathrm{~g}$ of protein (AOAC 990.03 method), $0.5 \mathrm{~g}$ of fat (CNS5036 method), $30.6 \mathrm{~g}$ of carbohydrate, $156 \mathrm{mg}$ of sodium (USEPA method 3051), $63.4 \mathrm{~g}$ of water (CNS5033 method), and $0.6 \mathrm{~g}$ of ash (CNS 5034 method). This ingredient analysis was validated by SGS Taiwan Ltd. Both the GMNs and PNs were provided free of charge by Nanchou Chemical Industrial Ltd. in Taiwan. In terms of calories, taste, and color of the placebo noodle was similar to glucomannan noodle; three expert testers confirmed similarity of the placebo and glucomannan noodle.

\subsection{Blood Biochemistry Analysis}

Blood samples from all subjects were measured after 12 hours of fasting at the beginning and after each 4-week intervention. Blood was obtained through capillary blood collection and blood sugar was examined instantly with the one-touch FreeStyle FREEDOM blood sugar monitor
(Abbott Diabetes Car Inc., CA, USA). Postprandial blood glucose was examined for each participant throughout the testing period. Other blood biomarkers, such as insulin, triglycerides, total cholesterol, alanine transaminase, $\mathrm{Cr}$, uric acid, and LDL-C or high density lipoprotein cholesterol (HDL-C) were analyzed from a $4 \mathrm{~mL}$ blood sample by using the Beckman Synchron LX-20 (Beckman Coulter Inc., CA, USA). Glycated hemoglobin (HbA1c) was analyzed from another $1.5 \mathrm{~mL}$ blood sample by using the Spotchem SP-4410 (Arkray Inc., Kyoto, Japan). All the experiments and procedures listed were performed in the authorized Yea-Tong laboratory center, in Chung-Li City, Taiwan.

\subsection{Determination of Plasma Vitamins A and $E$ and $\beta$-carotene}

The HPLC method for measuring vitamins $\mathrm{A}$ and $\mathrm{E}$ and $\beta$-carotene was performed according to Gueguen et al. with slight modifications. [20] Stock solutions of retinol, $\alpha$-tocopherol, and their corresponding internal standards (acetate form) were prepared in ethanol with BHT (2, 6-di-tert-butyl-4-methyl phenol) added. All handling 
operations were carried out in darkness and the plasma samples were rapidly thawed at room temperature. The plasma samples were mixed with internal standard, proteins were precipitated by ethanol, and extracted by n-hexane. The organic layer was evaporated and dissolved in the mobile phase for quantification of fat-soluble vitamins by HPLC (D-2000 Elite HPLC System; Hitachi Ltd., Tokyo, Japan). The quantities of vitamins A and $\mathrm{E}$ and $\beta$-carotene in each plasma sample were analyzed in triplicate.

\subsection{Statistical Analysis}

Statistical analyses were performed using SPSS 19 (SPSS Inc., Chicago, IL, USA). For intragroup tests, we performed a paired $t$ test with the Wilcoxon signed rank test to compare the differences of subjects' anthropometric measurements, clinical data, calorie and nutrient intake, biochemical characteristics, inflammatory markers, and antioxidant vitamins. For intergroup comparisons, we conducted an independent $t$ test with the Mann-Whitney $\mathrm{U}$ test to compare the final value or net present difference. All data were expressed as the means \pm standard deviations (SD). The results were considered to be statistically significant at $p<0.05$.

\section{Results}

\subsection{Dietary Treatment and Compliance}

In total, 32 MS subjects completed the study (Figure 1). There were no statistical differences in compliance between the groups and sequences. Both the GMN and PN preparations were generally well tolerated, although three patients from each group reported flatulence, increased gas production, and constipation in the first week. No other side effects or adverse events were reported by any of the participants. The calories, carbohydrate, protein, fat and dietary fiber intake analyses from $24 \mathrm{~h}$ food recalls showed all of the variables were not significantly different between the GMN and PN groups. (Table 2).

\subsection{Anthropometric Characteristics and Clinical Biochemistry}

All MS subjects $(n=32)$ in the GMN group received two servings of glucomannan noodles (400 g) daily, which were used to replace the staple carbohydrate in two meals. The placebo noodle group was given the same noodles without glucomannan. After the 4 weeks of the intervention, both the GMN and PN groups showed significantly decreased BW $(-0.79 \pm 1.17$ vs. $-0.58 \pm 1.08$ $\mathrm{kg})$, BMI $\left(-0.25 \pm 0.44\right.$ vs. $\left.-0.20 \pm 0.44 \mathrm{~kg} / \mathrm{m}^{2}\right)$, and waist circumference $(-1.86 \pm 2.55$ vs. $-0.98 \pm 1.99 \mathrm{~cm})$ as well as increased fat-soluble vitamin A $(0.47 \pm 1.18$ vs. $0.92 \pm$ $1.39 \mathrm{uM})$, vitamin $\mathrm{E}(4.73 \pm 13.25$ vs. $5.57 \pm 12.51 \mathrm{uM})$, and $\beta$-carotene $(0.79 \pm 1.68$ vs. $0.40 \pm 0.77 \mathrm{uM})$. Only the blood test for GPT (25.48 \pm 11.65 vs. $22.61 \pm 11.91 \mathrm{IU} / \mathrm{L}$, $\mathrm{p}=0.041$ ), high sensitivity C-reactive protein (hs-CRP) $(0.37 \pm 0.33$ v. $0.15 \pm 0.22 \mathrm{mg} / \mathrm{dL}, \mathrm{p}<0.001)$ were statistically significant in GMN. After analysis of the patients with T2DM (n = 24) according to physician clinical diagnosis, only the GMN group had significantly decreased BW (74.42 \pm 8.00 vs. $73.71 \pm 7.73 \mathrm{~kg}$, $p=0.008)$, BMI (28.02 \pm 3.19 vs. $27.78 \pm 3.22 \mathrm{~kg} / \mathrm{m}^{2}$, $p=0.011)$, waist circumference (92.08 \pm 6.04 vs. $90.17 \pm$ $6.51 \mathrm{~cm}, p<0.001)$, HbA1c $(8.22 \% \pm 1.53 \%$ vs. $7.59 \% \pm$ $1.49 \%, p=0.014)$, GPT (25.38 \pm 11.37 vs. $21.46 \pm 8.10$ $\mathrm{IU} / \mathrm{L}, p=0.016)$, and hs-CRP (0.42 \pm 0.34 vs. $0.16 \pm 0.25$ $\mathrm{mg} / \mathrm{dL}, p<0.001)$ after the 4 weeks of intervention. Interestingly, the change from baseline to end between GMN and PN groups, there were also statistically significant changes in waist circumference $(-1.92 \pm 2.23$ vs. $-0.48 \pm 1.61 \mathrm{~cm}, p=0.014)$, HbA1c $(-0.63 \% \pm 1.15 \%$ vs. $-0.07 \% \pm 0.61 \%, p=0.041)$, and hs-CRP $(-0.25 \pm$ 0.24 vs. $0.07 \pm 0.23 \mathrm{mg} / \mathrm{dL}, p=0.026$ ) between the GMN and $\mathrm{PN}$ groups according to an independent samples $t$ test (Table 4).

\section{Discussion}

Water-soluble dietary fiber has the properties of viscosity, gel formation, and expansion in size after consumption, which can promote satiety, delay gastric emptying, and decrease small bowel transit time; for these reasons, it is recommended by most diabetes and nutritional associations. [6] In our preclinical trial for oral glucose tolerance tests in ten normal subjects, the average glycemic index was $33.7 \pm 7.6$ for GMNs and 77.0 \pm 13.1 for PNs (Table 1). Eating low glucose food and low glycemic index diets were beneficial for weight loss and health management in subjects with T2DM. [21] Therefore, all subjects received two servings of noodles (400 g) daily, with or without glucomannan, to replace the staple carbohydrate in two meals while otherwise maintaining normal eating habits. Dietary Reference Intakes recommend consuming $14 \mathrm{~g}$ of DF per $1000 \mathrm{kcal}$, or $25 \mathrm{~g}$ for adult women and $38 \mathrm{~g}$ for adult men, to prevent chronic disease. [22] In the present study, both the GMN and PN groups had the same fiber intake at the baseline for MS subjects after four weeks intervention. It implies food processing by adding root fiber such as konjacderived glucomannan presents a possible option for physiological effectiveness against MS. According to a study by Doi et al., Japanese patients complained of abdominal pain, diarrhea, or other problems when consuming amounts of glucomannan greater than $5.2 \mathrm{~g}$. [11] In the present clinical trial in Taiwan, only $4 \mathrm{~g}$ of glucomannan was administered to the GMN group per day with significant decreases in BW, BMI, waist circumference, and hs-CRP subsequently observed not only in MS but also in T2DM individuals. Additionally, the 24 T2DM had significantly decreased HbA1c by 7.7\% after GMN intervention. Similar result was found in a randomized controlled metabolic trial showed that konjac-fiber-enriched test biscuits can improve glycemic and related factors for coronary heart disease in T2DM. [26] The unique fiber of the GMNs uniquely ameliorated glycemia in T2DM patients, as in Vuksan's study. [24] In a double-blind placebo-controlled crossover study after konjac-based glucomannan intervention, subjects were administered 3.9 g glucomannan capsules daily for 4 weeks that significantly reduced total cholesterol, LDL-C, triglycerides, and systolic blood pressure. [23] Based on 
the fat index analysis of this study, triglyceride, LDL-C, HDL-C and TC without statistical differences between GMN and PN consumption, and there is also no difference between the changes from baseline to end during GMN and PN consumption.

The laboratory and clinical evidence demonstrates a strong relationship between CRP and various MS phenomena. [24] In a study conducted by Salas-Salvado et al., $3 \mathrm{~g}$ of Plantago ovate husks and $1 \mathrm{~g}$ of glucomannan twice or three times daily in overweight or obese patients were found to be beneficial to weight loss and decreased LDL-C concentrations in plasma, but produced no difference in relation to glucose tolerance and serum CRP concentrations. [25] In the present study, a novel finding is that not only MS but also T2DM patients exhibited a significant decrease in hs-CRP after 4 weeks of the GMN intervention. Although we did not find any association between GMN to dietary fiber intake and lipid profile change in the current study, which is in contrast to other studies due to the intervention period may have been too short or insufficient glucomannan content in noodles.
Table 1. Nutrition facts and glycemic indices of GMNs and PNs per $100 \mathrm{~g}$

\begin{tabular}{lcc}
\hline & Glucomannan noodle & Placebo noodle \\
\hline Ingredients & & \\
Konjac glucomannan (g) & 1 & 0 \\
Calories (kcal) & 159 & 160 \\
Total fat (g) & 0.5 & 0.5 \\
Saturated Fat (g) & 0 & 0.2 \\
Trans Fat (g) & 0 & 0 \\
Sodium (mg) & 156 & 182 \\
Total carbohydrate (g) & 30.6 & 31.1 \\
Sugars (g) & 0 & 0 \\
Protein (g) & 7.9 & 7.8 \\
Water (g) & 63.4 & 59.9 \\
Ash (g) & 0.6 & 0.7 \\
Glycemic index & $33.7 \pm 7.6$ & $77.0 \pm 13.1$
\end{tabular}

GMN, glucomannan-containing noodle. PN, placebo noodle.

Table 2. Comparison between the mean and standard error of calories, macro nutrients dietary fiber intake from week 0 to week 4 in MS subjects

\begin{tabular}{|c|c|c|c|c|}
\hline \multirow[b]{2}{*}{ Variable } & \multicolumn{2}{|c|}{ Glucomannan noodle } & \multicolumn{2}{|c|}{ Placebo noodle } \\
\hline & Baseline & End & Baseline & End \\
\hline Calories (Kcal/day) & $1676.7 \pm 315.6$ & $1747.6 \pm 405.4$ & $1609.9 \pm 188.1$ & $1758.9 \pm 272.1$ \\
\hline Carbohydrate (g/day) & $228.2 \pm 55.7$ & $247.9 \pm 59.5$ & $209.5 \pm 46.1$ & $221.0 \pm 50.8$ \\
\hline Protein (g/day) & $59.5 \pm 14.0$ & $63.4 \pm 13.8$ & $55.8 \pm 13.4$ & $60.9 \pm 15.6$ \\
\hline Fat (g/day) & $67.6 \pm 16.2$ & $69.1 \pm 19.0$ & $66.2 \pm 13.4$ & $68.4 \pm 13.2$ \\
\hline Dietary fiber (g/day) & $12.7 \pm 5.6$ & $13.5 \pm 4.8$ & $11.9 \pm 4.7$ & $13.1 \pm 4.3$ \\
\hline
\end{tabular}

Table 3. Anthropometric measurement, biochemical characteristics of MS subjects from week 0 to week 4 in MS subjects

\begin{tabular}{|c|c|c|c|c|c|c|}
\hline \multirow[b]{2}{*}{ Variable } & \multicolumn{3}{|c|}{ Glucomannan noodle } & \multicolumn{3}{|c|}{ Placebo noodle } \\
\hline & Baseline & End & Changes & Baseline & End & Changes \\
\hline $\mathrm{BW}(\mathrm{kg})$ & $75.20 \pm 10.86$ & $74.41 \pm 10.49 *$ & $-0.79 \pm 1.17$ & $74.97 \pm 11.44$ & $74.39 \pm 11.27 *$ & $-0.58 \pm 1.08$ \\
\hline $\operatorname{BMI}\left(\mathrm{kg} / \mathrm{m}^{2}\right)$ & $28.77 \pm 3.83$ & $28.52 \pm 3.85^{*}$ & $-0.25 \pm 0.44$ & $28.71 \pm 4.10$ & $28.51 \pm 4.02 *$ & $-0.20 \pm 0.44$ \\
\hline Waist (cm) & $92.03 \pm 6.69$ & $90.17 \pm 6.80^{*}$ & $-1.86 \pm 2.55$ & $91.14 \pm 7.26$ & $90.15 \pm 7.11^{*}$ & $-0.98 \pm 1.99$ \\
\hline Waist-hip ratio & $0.91 \pm 0.05$ & $0.90 \pm 0.05$ & $-0.01 \pm 0.03$ & $0.90 \pm 0.05$ & $0.90 \pm 0.06$ & $0.00 \pm 0.03$ \\
\hline SBP (mm Hg) & $138.15 \pm 13.16$ & $137.79 \pm 9.92$ & $-0.36 \pm 11.79$ & $136.48 \pm 9.64$ & $134.18 \pm 11.43$ & $-2.30 \pm 8.15$ \\
\hline DBP (mm Hg) & $86.21 \pm 9.28$ & $84.30 \pm 8.32$ & $-1.91 \pm 7.85$ & $84.21 \pm 8.86$ & $85.39 \pm 8.87$ & $1.18 \pm 7.66$ \\
\hline FBG (mg/dL) & $163.12 \pm 62.25$ & $156.76 \pm 59.63$ & $-6.39 \pm 30.97$ & $171.03 \pm 64.07$ & $160.18 \pm 58.44$ & $-10.85 \pm 36.38$ \\
\hline HbA1c (\%) & $7.28 \pm 1.65$ & $7.08 \pm 1.54$ & $-0.21 \pm 0.73$ & $7.21 \pm 1.69$ & $7.12 \pm 1.60$ & $-0.08 \pm 0.55$ \\
\hline Insulin $(\mu \mathrm{U} / \mathrm{mL})$ & $12.23 \pm 10.15$ & $11.92 \pm 8.09$ & $-0.31 \pm 6.81$ & $19.43 \pm 28.31$ & $12.09 \pm 7.07$ & $-7.33 \pm 27.35$ \\
\hline HOMA-IR & $4.51 \pm 3.27$ & $4.17 \pm 2.51$ & $-0.34 \pm 2.52$ & $7.39 \pm 11.87$ & $4.56 \pm 2.73$ & $-2.83 \pm 12.28$ \\
\hline$\beta$-function (\% ) & $66.87 \pm 67.81$ & $78.46 \pm 96.75$ & $11.59 \pm 58.96$ & $110.02 \pm 206.25$ & $61.94 \pm 46.76$ & $-48.08 \pm 182.82$ \\
\hline TG (mg/dL) & $180.82 \pm 105.01$ & $199.70 \pm 138.07$ & $18.88 \pm 111.13$ & $195.45 \pm 108.82$ & $208.24 \pm 115.10$ & $12.79 \pm 69.67$ \\
\hline LDL-C (mg/dL) & $133.86 \pm 32.54$ & $137.24 \pm 33.99$ & $3.38 \pm 27.86$ & $135.39 \pm 28.02$ & $130.53 \pm 33.63$ & $-4.86 \pm 23.41$ \\
\hline HDL-C (mg/dL) & $45.19 \pm 11.06$ & $45.69 \pm 11.41$ & $0.50 \pm 4.73$ & $45.87 \pm 9.98$ & $45.98 \pm 11.41$ & $0.11 \pm 4.91$ \\
\hline TC (mg/dL) & $205.70 \pm 46.99$ & $202.36 \pm 46.84$ & $-3.33 \pm 30.21$ & $206.15 \pm 42.72$ & $204.91 \pm 34.60$ & $-1.24 \pm 37.38$ \\
\hline GPT (IU/L) & $25.48 \pm 11.65$ & $22.61 \pm 11.91^{*}$ & $-2.88 \pm 7.79$ & $24.88 \pm 13.74$ & $23.48 \pm 13.68$ & $-1.39 \pm 5.43$ \\
\hline Creatine (mg/dL) & $0.82 \pm 0.24$ & $0.83 \pm 0.25$ & $0.00 \pm 0.12$ & $0.81 \pm 0.27$ & $0.81 \pm 0.25$ & $0.00 \pm 0.11$ \\
\hline Uric acid (mg/dL) & $6.59 \pm 1.36$ & $6.57 \pm 1.77$ & $-0.03 \pm 1.31$ & $6.25 \pm 1.37$ & $6.32 \pm 1.06$ & $0.07 \pm 0.95$ \\
\hline Urea nitrogen (mg/dL) & $711.03 \pm 383.91$ & $784.48 \pm 368.08$ & $-73.45 \pm 410.43$ & $711.78 \pm 385.68$ & $683.10 \pm 326.62$ & $-48.68 \pm 324.05$ \\
\hline hs-CRP (mg/dL) & $0.37 \pm 0.33$ & $0.15 \pm 0.22 *$ & $-0.22 \pm 0.25$ & $0.35 \pm 0.51$ & $0.32 \pm 0.49$ & $-0.03 \pm 0.68$ \\
\hline Vitamin A $(\mu \mathrm{mol} / \mathrm{L})$ & $1.44 \pm 1.18$ & $1.90 \pm 0.64^{*}$ & $0.47 \pm 1.18$ & $1.06 \pm 0.91$ & $1.98 \pm 0.90 *$ & $0.92 \pm 1.39$ \\
\hline Vitamin E $(\mu \mathrm{mol} / \mathrm{L})$ & $13.04 \pm 9.09$ & $17.77 \pm 7.06^{*}$ & $4.73 \pm 13.25$ & $13.04 \pm 12.67$ & $18.61 \pm 6.29 *$ & $5.57 \pm 12.51$ \\
\hline$\beta$-carotene $(\mu \mathrm{mol} / \mathrm{L})$ & $0.68 \pm 0.48$ & $1.47 \pm 1.60^{*}$ & $0.79 \pm 1.68$ & $0.78 \pm 0.86$ & $1.17 \pm 0.92 *$ & $0.40 \pm 0.77$ \\
\hline
\end{tabular}

1. Data are presented as mean $\pm \mathrm{SD}$.

2. Values are significantly different after intervention within the group by paired t-test analysis $(* \mathrm{p}<0.05)$ and between the group by independent $t$-test $\left({ }^{\#} \mathrm{p}<0.05\right)$ 
Table 4. Anthropometric measurement, biochemical characteristics of MS subjects from week 0 to week 4 in T2DM subjects

\begin{tabular}{|c|c|c|c|c|c|c|}
\hline \multirow[b]{2}{*}{ Variable } & \multicolumn{3}{|c|}{ Glucomannan noodle } & \multicolumn{3}{|c|}{ Placebo noodle } \\
\hline & Baseline & End & Changes & Baseline & End & Changes \\
\hline BW (kg ) & $74.42 \pm 8.00$ & $73.71 \pm 7.73 *$ & $-0.71 \pm 1.20$ & $74.02 \pm 8.30$ & $73.73 \pm 8.36$ & $-0.29 \pm 0.85$ \\
\hline BMI $\left(\mathrm{kg} / \mathrm{m}^{2}\right)$ & $28.02 \pm 3.19$ & $27.78 \pm 3.22 *$ & $-0.24 \pm 0.43$ & $27.87 \pm 3.36$ & $27.80 \pm 3.44$ & $-0.07 \pm 0.32$ \\
\hline Waist (cm) & $92.08 \pm 6.04$ & $90.17 \pm 6.51^{*}$ & $-1.92 \pm 2.23^{\#}$ & $90.69 \pm 6.49$ & $90.21 \pm 6.55$ & $-0.48 \pm 1.61$ \\
\hline Waist-hip ratio & $0.92 \pm 0.04$ & $0.91 \pm 0.05$ & $-0.01 \pm 0.03$ & $0.91 \pm 0.05$ & $0.91 \pm 0.06$ & $0.00 \pm 0.03$ \\
\hline SBP (mm Hg) & $137.92 \pm 12.95$ & $139.67 \pm 9.37$ & $1.75 \pm 11.96$ & $138.54 \pm 9.36$ & $135.83 \pm 12.01$ & $-2.71 \pm 9.06$ \\
\hline DBP (mm Hg) & $86.63 \pm 8.21$ & $86.21 \pm 7.47$ & $-0.42 \pm 6.01$ & $86.04 \pm 8.94$ & $86.92 \pm 8.70$ & $0.88 \pm 8.07$ \\
\hline FBG (mg/dL) & $182.17 \pm 63.08$ & $174.63 \pm 60.57$ & $-7.54 \pm 36.12$ & $190.58 \pm 63.35$ & $179.29 \pm 57.74$ & $-11.29 \pm 39.99$ \\
\hline HbA1c (\%) & $8.22 \pm 1.53$ & $7.59 \pm 1.49 *$ & $-0.63 \pm 1.15^{\#}$ & $7.76 \pm 1.64$ & $7.69 \pm 1.52$ & $-0.07 \pm 0.61$ \\
\hline Insulin $(\mu \mathrm{U} / \mathrm{mL})$ & $10.81 \pm 9.27$ & $10.11 \pm 6.08$ & $-0.70 \pm 6.89$ & $11.54 \pm 8.14$ & $12.06 \pm 7.79$ & $0.52 \pm 6.30$ \\
\hline HOMA-IR & $4.53 \pm 3.32$ & $4.00 \pm 1.98$ & $-0.53 \pm 2.73$ & $4.79 \pm 2.17$ & $5.04 \pm 2.97$ & $0.25 \pm 3.00$ \\
\hline$\beta$-function (\% ) & $47.66 \pm 48.28$ & $51.59 \pm 64.23$ & $3.93 \pm 56.27$ & $51.02 \pm 63.01$ & $49.21 \pm 42.37$ & $-1.81 \pm 34.10$ \\
\hline LDL-C (mg/dL) & $135.28 \pm 35.24$ & $134.60 \pm 35.49$ & $-0.68 \pm 20.60$ & $132.56 \pm 30.11$ & $131.40 \pm 32.07$ & $-1.16 \pm 15.36$ \\
\hline HDL-C (mg/dL) & $45.35 \pm 11.52$ & $45.35 \pm 12.07$ & $-0.01 \pm 5.02$ & $46.10 \pm 10.70$ & $45.63 \pm 12.13$ & $-0.47 \pm 4.63$ \\
\hline $\mathrm{TC}(\mathrm{mg} / \mathrm{dL})$ & $217.29 \pm 33.24$ & $212.33 \pm 32.71$ & $-4.96 \pm 23.42$ & $213.29 \pm 30.60$ & $210.46 \pm 29.41$ & $-2.83 \pm 15.70$ \\
\hline GPT (IU/L) & $25.38 \pm 11.37$ & $21.46 \pm 8.10^{*}$ & $-3.92 \pm 7.36$ & $22.42 \pm 10.16$ & $21.08 \pm 9.08$ & $-1.33 \pm 4.08$ \\
\hline Creatine (mg/dL) & $0.86 \pm 0.25$ & $0.88 \pm 0.26$ & $0.02 \pm 0.11$ & $0.85 \pm 0.29$ & $0.85 \pm 0.28$ & $0.00 \pm 0.11$ \\
\hline Uric acid (mg/dL) & $6.58 \pm 1.30$ & $6.72 \pm 1.88$ & $0.13 \pm 1.49$ & $6.34 \pm 1.43$ & $6.44 \pm 1.06$ & $0.10 \pm 1.05$ \\
\hline Urea nitrogen (mg/dL) & $678.88 \pm 309.74$ & $754.79 \pm 384.78$ & $75.91 \pm 382.01$ & $683.26 \pm 315.68$ & $678.74 \pm 321.40$ & $-4.52 \pm 308.82$ \\
\hline hs-CRP (mg/dL) & $0.42 \pm 0.34$ & $0.16 \pm 0.25^{*}$ & $-0.25 \pm 0.24^{\#}$ & $0.24 \pm 0.30$ & $0.31 \pm 0.54$ & $0.07 \pm 0.23$ \\
\hline Vitamin A $(\mu \mathrm{mol} / \mathrm{L})$ & $1.67 \pm 1.23$ & $2.06 \pm 0.60$ & $0.39 \pm 1.23$ & $1.09 \pm 1.00$ & $2.17 \pm 0.97^{*}$ & $1.08 \pm 1.56$ \\
\hline Vitamin E $(\mu \mathrm{mol} / \mathrm{L})$ & $14.89 \pm 9.07$ & $19.45 \pm 6.92$ & $4.56 \pm 13.94$ & $13.09 \pm 14.12$ & $19.99 \pm 6.42^{*}$ & $6.90 \pm 13.66$ \\
\hline$\beta$-carotene $(\mu \mathrm{mol} / \mathrm{L})$ & $0.73 \pm 0.50$ & $1.53 \pm 1.68^{*}$ & $0.80 \pm 1.76$ & $0.71 \pm 0.85$ & $1.14 \pm 1.00$ & $0.43 \pm 0.75$ \\
\hline
\end{tabular}

1. Data are presented as mean $\pm \mathrm{SD}$.

2. Values are significantly different after intervention within the group by paired t-test analysis $\left({ }^{*} \mathrm{p}<0.05\right)$ and between the group by independent $t$-test $(\# \mathrm{p}<0.05)$.

Soluble DF in the gut could attenuate the absorption of lipid-soluble compounds. [26] In the present study, fatsoluble vitamins such as vitamin $A$, vitamin $E$, and $\beta$ carotene were maintained in the normal range during the GMN intervention after 1 month. An animal study by Wu et al. demonstrated that konjac-derived glucomannan could modulate antioxidant defenses in rats fed a high-fat diet through enhanced gene expression of glutathione peroxidase, catalase, and superoxide dismutase in the liver, as well as elevated $\alpha$-tocopherol in the blood. [27] Excessive oxidative stress can elicit intracellular downregulation in signals of insulin action, and antioxidants can provide protection against oxidative stress through neutralization of intracellular reactive oxygen species. [28] The clinical antioxidant profiles and redox imbalance in subjects may be determined after we do more work and obtain more data in the future. In summary, the GMN supplementation for a 4-week period can reduce the risk factors of MS, and can decrease the oxidative stress inside the body, especially in subjects concomitant with T2DM. Using GMNs to substitute for the major carbohydrate would be convenient in MS patients and long-term studies on the effect of GMNs on glycemic profile and inflammatory markers in metabolic syndrome are necessary.

\section{Acknowledgments}

The authors thank Yun-Fan Lu (Sherry Lu), a master's graduate from the Australian National University, for her assistance with proofreading and editing this manuscript. Funding support by 96TMU-WFH-16 and NSC-1012313-B-038-004.

\section{Conflict of Interest}

Each one and all authors declare no conflict of interest.

\section{Abbreviations}

Cr, creatine; DF, dietary fiber; GMN, glucomannan noodle; HbA1c, glycated hemoglobin; HDL-C, high density lipoprotein cholesterol; HOMA-IR, the homeostatic model assessment; hs-CRP, high sensitivity C-reactive protein; LDL-C, low density lipoprotein cholesterol; PN, placebo noodle; T2DM, type 2 diabetes mellitus; TC, total cholesterol; TG, total triglycerides

\section{References}

[1] Li G, de Courten M, Jiao S, Wang Y (2010). Prevalence and characteristics of the metabolic syndrome among adults in Beijing, China. Asia Pacific journal of clinical nutrition 19 (1): 98-102.

[2] Huang KC, Lee LT, Chen CY, Sung PK (2008). All-cause and cardiovascular disease mortality increased with metabolic syndrome in Taiwanese. Obesity 16 (3): 684-689.

[3] Paoletti R, Bolego C, Poli A, Cignarella A (2006). Metabolic syndrome, inflammation and atherosclerosis. Vascular health and risk management 2 (2): 145-152. 
[4] Grundy SM, Cleeman JI, Daniels SR, Donato KA, Eckel RH, Franklin BA, Gordon DJ, Krauss RM, Savage PJ, Smith SC, Jr., Spertus JA, Costa F (2005). Diagnosis and management of the metabolic syndrome: an American Heart Association/National Heart, Lung, and Blood Institute Scientific Statement. Circulation 112 (17): 2735-2752.

[5] Grundy SM (2007). Metabolic syndrome: a multiplex cardiovascular risk factor. The Journal of clinical endocrinology and metabolism 92 (2): 399-404.

[6] Weickert MO, Pfeiffer AF (2008). Metabolic effects of dietary fiber consumption and prevention of diabetes. The Journal of nutrition 138 (3): 439-442

[7] Steemburgo T, Dall'Alba V, Almeida JC, Zelmanovitz T, Gross JL, de Azevedo MJ (2009). Intake of soluble fibers has a protective role for the presence of metabolic syndrome in patients with type 2 diabetes. European journal of clinical nutrition 63 (1): 127-133.

[8] Bantle JP, Wylie-Rosett J, Albright AL, Apovian CM, Clark NG, Franz MJ, Hoogwerf BJ, Lichtenstein AH, Mayer-Davis E, Mooradian AD, Wheeler ML (2008). Nutrition recommendations and interventions for diabetes: a position statement of the American Diabetes Association. Diabetes care 31 Suppl 1: S61-78.

[9] Weng LC, Lee NJ, Yeh WT, Ho LT, Pan WH (2012). Lower intake of magnesium and dietary fiber increases the incidence of type 2 diabetes in Taiwanese. Journal of the Formosan Medical Association = Taiwan yi zhi 111 (11): 651-659.

[10] Chua M, Baldwin TC, Hocking TJ, Chan K (2010). Traditional uses and potential health benefits of Amorphophallus konjac K. Koch ex N.E.Br. Journal of ethnopharmacology 128 (2): 268-278.

[11] Doi K (1995). Effect of konjac fibre (glucomannan) on glucose and lipids. European journal of clinical nutrition 49 Suppl 3 : S190-197.

[12] Chen HL, Cheng HC, Wu WT, Liu YJ, Liu SY (2008). Supplementation of konjac glucomannan into a low-fiber Chinese diet promoted bowel movement and improved colonic ecology in constipated adults: a placebo-controlled, diet-controlled trial. Journal of the American College of Nutrition 27 (1): 102-108.

[13] Yoshida M, Vanstone CA, Parsons WD, Zawistowski J, Jones PJ (2006). Effect of plant sterols and glucomannan on lipids in individuals with and without type II diabetes. European journal of clinical nutrition 60 (4):529-537.

[14] Zalewski BM, Chmielewska A, Szajewska H (2015). The effect of glucomannan on body weight in overweight or obese children and adults: a systematic review of randomized controlled trials. Nutrition 31 (3):437-442 e432.

[15] Loening-Baucke V, Miele E, Staiano A (2004) Fiber (glucomannan) is beneficial in the treatment of childhood constipation. Pediatrics 113 (3 Pt 1): e259-264.

[16] Al-Ghazzewi FH, Tester RF (2012) Efficacy of cellulase and mannanase hydrolysates of konjac glucomannan to promote the growth of lactic acid bacteria. J Sci Food Agric 92 (11): 2394-2396.

[17] Sood N, Baker WL, Coleman CI (2008). Effect of glucomannan on plasma lipid and glucose concentrations, body weight, and blood pressure: systematic review and meta-analysis. Am J Clin Nutr 88 (4): 1167-1175.

[18] Chen HL, Sheu WH, Tai TS, Liaw YP, Chen YC (2003). Konjac supplement alleviated hypercholesterolemia and hyperglycemia in type 2 diabetic subjects--a randomized double-blind trial. Journal of the American College of Nutrition 22 (1): 36-42

[19] Grundy SM, Cleeman JI, Merz CN, Brewer HB, Jr., Clark LT, Hunninghake DB, Pasternak RC, Smith SC, Jr., Stone NJ, National Heart L, Blood I, American College of Cardiology F, American Heart A (2004). Implications of recent clinical trials for the National Cholesterol Education Program Adult Treatment Panel III guidelines. Circulation 110 (2): 227-239.

[20] Gueguen S, Herbeth B, Siest G, Leroy P (2002). An isocratic liquid chromatographic method with diode-array detection for the simultaneous determination of alpha-tocopherol, retinol, and five carotenoids in human serum. Journal of chromatographic science 40 (2): 69-76.

[21] McGeoch SC, Holtrop G, Fyfe C, Lobley GE, Pearson DW, Abraham P, Megson IL, Macrury SM, Johnstone AM (2011). Food intake and dietary glycaemic index in free-living adults with and without type 2 diabetes mellitus. Nutrients 3 (6): 683-693.

[22] Slavin JL (2008). Position of the American Dietetic Association: health implications of dietary fiber. J Am Diet Assoc 108 (10): 1716-1731.

[23] Arvill A, Bodin L (1995). Effect of short-term ingestion of konjac glucomannan on serum cholesterol in healthy men. Am J Clin Nutr 61 (3): 585-589

[24] Devaraj S, Singh U, Jialal I (2009). Human C-reactive protein and the metabolic syndrome. Current opinion in lipidology 20 (3): 182-189.

[25] Salas-Salvado J, Farres X, Luque X, Narejos S, Borrell M, Basora J, Anguera A, Torres F, Bullo M, Balanza R, Fiber in Obesity-Study G (2008). Effect of two doses of a mixture of soluble fibres on body weight and metabolic variables in overweight or obese patients: a randomised trial. The British journal of nutrition 99 (6): 1380-1387.

[26] Palafox-Carlos H, Ayala-Zavala JF, Gonzalez-Aguilar GA (2011) The role of dietary fiber in the bioaccessibility and bioavailability of fruit and vegetable antioxidants. J Food Sci 76 (1): R6-R15.

[27] Wu WT, Chen HL (2011) Konjac glucomannan and inulin systematically modulate antioxidant defense in rats fed a high-fat fiber-free diet. J Agric Food Chem 59 (17): 9194-9200.

[28] Evans JL (2007) Antioxidants: do they have a role in the treatment of insulin resistance? The Indian journal of medical research 125 (3): 355-372. 